BULLETIN OF THE POLISH

ACADEMY OF SCIENCES

MATHEMATICS

Vol. 58, No. 3, 2010

\title{
A Few Remarks on the Mohan Kumar Theorem
}

by

\author{
Zbigniew JELONEK \\ Presented by Andrzej SKOWROŃSKI
}

Summary. We give a simple geometric proof of Mohan Kumar's result about complete intersections.

1. Introduction. Let $k$ be an algebraically closed field. The aim of this note is to give a simple proof of the following:

TheOREM 1.1. Let $X \subset k^{n}$ be a smooth algebraic variety and $2 \operatorname{dim} X+$ $2 \leq n$. Then $\mu\left(I(X) / I(X)^{2}\right)=\mu(I(X))$, where $\mu(I)$ denotes the minimal number of generators of a $k\left[x_{1}, \ldots, x_{n}\right]$-module $I$.

COROLlary 1.2. Let $X \subset k^{n}$ be a smooth algebraic variety and $2 \operatorname{dim} X$ $+2 \leq n$. Then $X$ is a complete intersection if and only if the tangent bundle of $X$ is trivial.

Here $I(X)$ denotes the ideal of the variety $X$. Let us recall also that a variety $X \subset k^{n}$ is a complete intersection if $\mu(I(X))=\operatorname{codim} X$.

Theorem 1.1 is a "weak" version of the more general result of Mohan Kumar [2]. However our proof has a clear geometric nature and it is very short.

2. Main result. We start with the following:

Lemma 2.1. Let $X$ be a smooth affine variety. Let $\mathbf{F}$ be an algebraic vector bundle on $X$ and $\mu(\mathbf{F})=p>\operatorname{dim} X$. If $\mathbf{s} \in \Gamma(X, \mathbf{F})$ is a nowhere vanishing section, then there are sections $\mathbf{t}_{i} \in \Gamma(X, \mathbf{F}), i=1, \ldots, p-1$, such that the sections $\mathbf{s}$ and $\mathbf{t}_{1}, \ldots, \mathbf{t}_{p-1}$ generate the bundle $\mathbf{F}$.

2010 Mathematics Subject Classification: 13C10, 14J99, 14R99.

Key words and phrases: algebraic vector bundle, complete intersection, unimodular row. 
Proof. Let $\mathbf{r}_{1}, \ldots, \mathbf{r}_{p} \in \Gamma(X, \mathbf{F})$ generate $\mathbf{F}$. Hence we have

$$
\mathbf{s}=\sum_{i=1}^{p} \alpha_{i} \mathbf{r}_{i},
$$

where $\alpha_{i} \in k[X]$. By assumption the row $\left(\alpha_{1}, \ldots, \alpha_{p}\right)$ is unimodular. Since $p>\operatorname{dim} X$ we have by the Suslin Cancellation Theorem (see [4]) that the row $\left(\alpha_{1}, \ldots, \alpha_{p}\right)$ can be completed to a matrix

$$
\left[\begin{array}{ccc}
\alpha_{1} & \ldots & \alpha_{p} \\
f_{1,1} & \ldots & f_{1, p} \\
\vdots & \ldots & \vdots \\
f_{p-1,1} & \ldots & f_{p-1, p}
\end{array}\right]
$$

with determinant 1 . Now it is enough to take $\mathbf{t}_{i}=\sum_{k=1}^{p} f_{i k} \mathbf{r}_{k}, i=1, \ldots$, $p-1$.

REMARK 2.2. If we assume that $\mu(\mathbf{F})>\operatorname{dim} X+1$ then we can use the weaker Bass Cancellation Theorem, instead of Suslin's result. In fact this weaker version is what we actually need.

Proof of Theorem 1.1. Now we are in a position to prove Theorem 1.1 . Take a sufficiently general system of coordinates and let $H=\left\{x: x_{n}=0\right\}$. Next consider the projection $\pi: k^{n} \ni\left(x_{1}, \ldots, x_{n}\right) \mapsto\left(x_{1}, \ldots, x_{n-1}, 0\right) \in$ $H \cong k^{n-1}$. It is easy to see that the projection $\pi$ restricted to $X$ is an embedding of $X$ into $H$. In particular there exists a polynomial $f$ such that on $X$ we have $x_{n}=f\left(x_{1}, \ldots, x_{n-1}\right)$. If we change variables by the automorphism

$$
\Phi\left(x_{1}, \ldots, x_{n}\right)=\left(x_{1}, \ldots, x_{n-1}, x_{n}-f\left(x_{1}, \ldots, x_{n-1}\right)\right)
$$

we can assume that $X \subset H$.

Let $\mathbf{F}=I(X) / I(X)^{2}$ be the conormal vector bundle of $X$. Note that the polynomial $x_{n}$ gives a nowhere vanishing section of this bundle. Indeed, locally $x_{n}$ is a part of a system of local parameters of $X$ and since local parameters locally are free generators of $\mathbf{F}$ we conclude that $x_{n}$ does not vanish at any point.

Let $p=\mu(\mathbf{F})$. By Lemma 2.1 we have polynomials $r_{1}, \ldots, r_{p-1}$ such that $\mathbf{F}$ is generated by the classes of the polynomials $x_{n}, r_{1}, \ldots, r_{p-1}$. In particular, the polynomials $x_{n}, r_{1}, \ldots, r_{p-1}$ locally describe $X$, i.e., $I(X)_{a}=$ $\left(x_{n}, r_{1}, \ldots, r_{p-1}\right)_{a}$ for $a$ from some open neighborhood of $X$. Moreover, we can assume that the polynomials $r_{i}, i=1, \ldots, p-1$, do not depend on the variable $x_{n}$. 
In an obvious manner $r_{1}, \ldots, r_{p-1}$ locally describe $X$ as a subvariety of $H=\left\{x: x_{n}=0\right\} \cong k^{n-1}$. Hence on $H$ we have

$$
V\left(r_{1}, \ldots, r_{p-1}\right)=X \cup Y,
$$

where $X \cap Y=\emptyset$. There is a function $h \in k[H]$ such that $h=0$ on $X$ and $h=1$ on $Y$. The function $h$ can be considered on the whole of $k^{n}$-it does not depend on $x_{n}$. Let us note that the row $\left(r_{1}, \ldots, r_{p-1}\right)$ is unimodular on $U:=$ $k^{n} \backslash\{x: h(h-1)=0\}$. This easily implies that the row $\left(r_{1}, \ldots, r_{p-1}, r_{p}\right)$, where $r_{p}=x_{n}$, is on $U$ equivalent to $(1,0, \ldots, 0)$ (see e.g. [1, Proposition 5.3, p. 44]). In particular there is a matrix $\mathbb{A} \in G L(p, k[U])$ whose first row is $\left(r_{1}, \ldots, r_{p}\right)$.

Now we can finish by the classical Serre construction. Take $V_{0}=\{x \in$ $\left.k^{n}: h(x) \neq 0\right\}$ and $V_{1}=\left\{x \in k^{n}: h(x) \neq 1\right\}$. We have two epimorphisms

$$
\Phi_{1}: \mathcal{O}_{V_{1}}^{p} \ni\left(h_{1}, \ldots, h_{p}\right) \mapsto \sum_{i=1}^{p} h_{i} r_{i} \in I(X) \quad \text { over } V_{1}
$$

and

$$
\Phi_{0}: \mathcal{O}_{V_{0}}^{p} \ni\left(h_{1}, \ldots, h_{p}\right) \rightarrow h_{1} \in I(X) \quad \text { over } V_{0} .
$$

On $U=V_{0} \cap V_{1}$ we have $\Phi_{1}=\Phi_{0} \circ \mathbb{A}$. Hence if we glue over $U$ two trivial vector bundles of rank $p$ on $V_{0}$ and $V_{1}$ using the matrix $\mathbb{A}$, we obtain an algebraic vector bundle $\mathbf{E}$ of rank $p$ and an epimorphism $\Phi: \mathbf{E} \rightarrow I(X)$. By the Quillen-Suslin Theorem (see [1], [3]) the vector bundle $\mathbf{E}$ is trivial, which concludes the proof.

Proof of Corollary 1.2. If $X$ is a complete intersection, then the conormal bundle of $X$ is trivial. Hence the normal bundle is trivial too. Now by the Suslin Cancellation Theorem (see 4]) we conclude that the tangent bundle of $X$ is trivial.

Conversely, if the tangent bundle of $X$ is trivial, then for the same reason the conormal bundle is, and we can use Theorem 1.1 .

Acknowledgments. The author was partially supported by the grant of Polish Ministry of Science 2010-2013.

\section{References}

[1] T. Lam, Serre Conjecture, Springer, New York, 1978.

[2] N. Mohan Kumar, On two conjectures about polynomial rings, Invent. Math. 46 (1978), 225-236.

[3] D. Quillen, Projective modules over polynomial rings, Invent. Math. 36 (1976), 167171.

[4] A. Suslin, A theorem on cancellation for projective modules over algebras, Dokl. Akad. Nauk SSSR 236 (1977), 808-811 (in Russian). 
Zbigniew Jelonek

Instytut Matematyczny PAN

Śniadeckich 8

00-956 Warszawa, Poland

E-mail: najelone@cyf-kr.edu.pl

Received September 30, 2010;

received in final form October 18, 2010

(7786) 